\title{
COMPLEMENTING SETS OF $n$-TUPLES OF INTEGERS
}

\author{
MELVYN B. NATHANSON ${ }^{1}$
}

\begin{abstract}
Let $S, A_{1}, A_{2}, \cdots, A_{p}$ be finite nonempty sets of $n$-tuples of integers such that, if $a_{i} \in A_{i}$ for $i=1,2, \cdots, p$, then $a_{1}+a_{2}+\cdots+a_{p} \in S$, and such that every $s \in S$ has a unique representation as a sum $s=a_{1}+a_{2}+\cdots+a_{p}$ with $a_{i} \in A_{i}$. If $S$ is the cartesian product of $n$ sets of integers, then each $A_{i}$ is also the cartesian product of $n$ sets of integers, and conversely.
\end{abstract}

Let $S, A_{1}, A_{2}, \cdots, A_{p}$ be sets of $n$-tuples of integers. Define addition of $n$-tuples componentwise. Then $S$ is the sum of $A_{1}, A_{2}, \cdots, A_{p}$, denoted $S=A_{1}+A_{2}+\cdots+A_{p}$, if $S=\left\{a_{1}+a_{2}+\cdots+a_{p} \mid a_{i} \in A_{i}\right.$ for $i=1$, $2, \cdots, p\}$. If $S$ is the sum of $A_{1}, A_{2}, \cdots, A_{p}$, and if for each $s \in S$ there exist unique $n$-tuples $a_{i} \in A_{i}$ such that $s=a_{1}+a_{2}+\cdots+a_{p}$, then $A_{1}$, $A_{2}, \cdots, A_{p}$ are called complementing sets for $S$, denoted $S \cong A_{1}+$ $A_{2}+\cdots+A_{p}$. A set of $n$-tuples is proper if it is the cartesian product of $n$ sets of integers. For positive integers $u$ and $v$, let $S=\{0,1,2, \cdots, u\} \times$ $\{0,1,2, \cdots, v\}$. If $A_{1}$ and $A_{2}$ are subsets of $S$ such that $S \cong A_{1}+A_{2}$, then Hansen [1] proved that $A_{1}$ and $A_{2}$ are proper. This result extends to the general case of arbitrary $n$ and $p$, and $S$ the cartesian product of any $n$ finite sets of integers.

THEOREM. Let $S, A_{1}, A_{2}, \cdots, A_{p}$ be finite nonempty sets of $n$-tuples of integers such that $S \cong A_{1}+A_{2}+\cdots+A_{p}$. Then $S$ is proper if and only if each $A_{i}$ is proper.

Proof. Suppose that $S$ is proper. By translation, it is enough to consider only the case in which all coordinates of all $n$-tuples of $S, A_{1}, \cdots, A_{p}$ are nonnegative integers. Let $Z\left[X_{1}, \cdots, X_{n}\right]$ be the ring of polynomials in $n$ variables with integral coefficients. Define $F, G_{1}, G_{2}, \cdots, G_{p}$ in $Z\left[X_{1}, \cdots, X_{n}\right]$ by

$$
\begin{aligned}
F & =\sum_{\left(s_{1}, s_{2}, \cdots, s_{n}\right) \in S} X_{1}^{s_{1}} X_{2}^{s_{2}} \cdots X_{n}^{s_{n}}, \\
G_{i} & =\sum_{\left(a_{1}, a_{2}, \cdots, a_{n}\right) \in A_{i}} X_{1}^{a_{1}} X_{2}^{a_{2}} \cdots X_{n}^{a_{n}} .
\end{aligned}
$$

Received by the editors July 30, 1971.

AMS 1970 subject classifications. Primary 10L05; Secondary 10A45, 10J99, 05 A15.

Key words and phrases. Complementing sets, sumsets of integers, addition of $n$ tuples of integers.

${ }^{1}$ Supported in part by an NSF Predoctoral Traineeship from the University of Rochester.

(c) American Mathematical Society 1972 
Since $S$ is proper, there exist finite sets of nonnegative integers $S_{1}, S_{2}, \cdots$, $S_{n}$ such that $S=S_{1} \times S_{2} \times \cdots \times S_{n}$. Then

$$
F=\prod_{j=1}^{n}\left(\sum_{s_{j} \in S_{j}} X_{j}^{s_{j}}\right) .
$$

The polynomial $F$ is the product of irreducible polynomials, and by (1), each of these irreducibles is a polynomial in exactly one variable. Each $G_{i}$ is also a product of irreducible polynomials. Since $A_{1}+A_{2}+\cdots+$ $A_{p} \cong S$, it follows that $F=G_{1} G_{2} \cdots G_{p}$. Since $Z\left[X_{1}, \cdots, X_{n}\right]$ is a unique factorization domain, each irreducible factor of each $G_{i}$ is a factor of $F$, and so is a polynomial in exactly one variable. Thus there exist polynomials $g_{i j} \in Z\left[X_{j}\right]$ for $i=1,2, \cdots, p$ and $j=1,2, \cdots, n$ such that $G_{i}=$ $g_{i 1} g_{i 2} \cdots g_{i n}$. Let $A_{i j}$ be the finite set of nonnegative integers which are the powers of $X_{j}$ occurring with nonzero coefficient in $g_{i j}$. Then $A_{i}=$ $A_{i 1} \times A_{i 2} \times \cdots \times A_{i n}$ for $i=1,2, \cdots, p$, and $A_{i}$ is proper. (Moreover, $A_{1 j}+A_{2 j}+\cdots+A_{p j} \cong S_{j}$ for $j=1,2, \cdots, n$.)

Conversely, suppose that each $A_{i}$ is proper. Then there exist finite sets of integers $A_{i j}$ for $i=1,2, \cdots, p$ and $j=1,2, \cdots, n$ such that $A_{i}=A_{i 1} \times$ $A_{i 2} \times \cdots \times A_{i n}$. Let $S_{j}=A_{1 j}+A_{2 j}+\cdots+A_{p j}$ for $j=1,2, \cdots, n$. Then $S=S_{1} \times S_{2} \times \cdots \times S_{n}$, and so $S$ is proper.

REMARK. The theorem is false if $S$ is the cartesian product of infinite sets. If $N$ is the set of nonnegative integers, there exist sets $A_{1}$ and $A_{2}$ which are not proper but satisfy $A_{1}+A_{2} \cong N \times N$. Hansen [1] and Niven [2] have determined all sets $A_{1}$ and $A_{2}$ such that $A_{1}+A_{2} \cong N \times N$.

\section{REFERENCES}

1. R. T. Hansen, Complementing pairs of subsets of the plane, Duke Math. J. 36 (1969), 441-449. MR 39 \#5719.

2. I. Niven, A characterization of complementing sets of pairs of integers, Duke Math. J. 38 (1971), 193-203. MR 43 \#179.

UNIVERSITY OF ROCHESTER, ROCHESTER, NEW YORK 14627

Current address: Southern Illinois University, Carbondale, Illinois 62901 